Abschließend sei noch erwähnt, daß man die hier durchgeführte Rechnung natürlich ebensogut für Löcher in einem fast besetzten Band wie für Elektronen in einem fast leeren Band hätte durchführen können. Man hat dann in (1) lediglich $n_{1 k}$ durch $1-\bar{n}_{2 k}$ und $n_{2 k}$ durch $1-\bar{n}_{1 k}$ zu ersetzen.

Da am oberen Bandrand $\varepsilon_{k}=-a k^{2}$ bis auf eine belanglose Konstante gesetzt werden kann $(a>0)$, wenn nicht besondere Kristallanisotropien vorliegen, haben die Energieniveaus des Systems wiederum die Form (1), woraus folgt, daß die Resultate die gleichen bleiben. Unübersichtlich wird die Situation nur, wenn ein Unterband fast leer, das andere fast besetzt ist. Dieser Fall wurde jedoch nicht betrachtet.

Herrn Prof. Dr. G. Heber danke ich herzlich für wertvolle Anregungen.

\title{
Zur Theorie des Ferromagnetismus II. Das Heisenberg-Modell
}

\author{
Von Klaus Meyer \\ Aus dem Theoretisch-Physikalischen Institut der Universität Jena \\ (Z. Naturforschg. 12 a, 797-804 [1957]; eingegangen am 13. Mai 1957)
}

\begin{abstract}
Es wird gezeigt, daß man die Energieniveaus des Heisenberg-Modells für ein Ferromagnetikum (Spin 1/2 pro Atom) durch Quasiteilchen darstellen kann, die der FERMI-Statistik gehorchen. Die thermodynamischen Zustandsgrößen des Systems werden unter Berücksichtigung der Wechselwirkung dieser Quasiteilchen berechnet. Die Resultate werden mit denen verglichen, die man erhält, wenn man das System für den Fall positiven Austauschintegrals durch wechselwirkungsfreie Quasiteilchen darstellt. Darüber hinaus wird angedeutet, was für Quasiteilchen zu erwarten sind, wenn mehr als ein „ferromagnetisches“ Elektron pro Atom vorhanden ist und gegebenenfalls Bahnmomente zu berücksichtigen sind.
\end{abstract}

Für die folgenden Untersuchungen wählen wir das Heitler-London-Modell für einen ferromagnetischen Kristall mit nur einem Valenzelektron pro Atom außerhalb abgeschlossener Schalen, das wir außerdem noch in der Näherung der Energieschwerpunktmethode behandeln werden. Dieses Modell wird gewöhnlich als Heisenberg-Modell bezeichnet. Natürlich bedeutet es eine starke Idealisierung der Wirklichkeit, ist aber andererseits genügend einfach gebaut, um den verschiedensten Näherungsmethoden leicht zugänglich zu sein.

Insbesondere soll hier die üblicherweise benutzte Molekularfeldmethode einer Analyse unterzogen werden, und zwar unter besonderer Berücksichtigung der Tatsache, daß man die angeregten Zustände des betrachteten Modells durch gewisse FerMI-Quasiteilchen darstellen kann, wie dies in allgemeinerer Form schon früher ${ }^{1,2}$ gezeigt worden ist. Der Beweis dafür wird in diesem gegenüber der früheren Arbeit ${ }^{2}$ vereinfachten Fall nochmals in anderer Weise durchgeführt werden.

1 D. FRANK, Z. Phys. 146, 615 [1956].

2 K. Meyer, Z. Naturforschg. 11 a, 865 [1956].
Dabei zeigt sich, daß auch die Langevinsche Theorie des Paramagnetismus in ihrer an die Quantentheorie angepaßten Form als eine Art Spinwellentheorie interpretiert werden kann. Andererseits soll überprüft werden, welche Resultate man erhält und wie weit diese brauchbar sind, wenn man das System genähert durch wechselwirkungsfreie Quasiteilchen darstellt (vgl. Anm. ${ }^{3}$ ).

\section{Das Modell}

Das hier benutzte Modell wird beschrieben durch den Diracschen Austauschoperator

$$
\mathfrak{\mathfrak { D }}=2 H \mu_{\mathrm{B}} \sum_{\mathfrak{n}} \mathfrak{\Im}_{\mathfrak{n}}^{z}-\sum_{\mathfrak{n}, \mathfrak{m}} I_{\mathfrak{n}, \mathfrak{m}} \Im_{\mathfrak{n}} \Im_{\mathfrak{m}} .
$$

$H$ ist das äußere Magnetfeld, $\mu_{\mathrm{B}}$ das вoHrsche Magneton, $\mathfrak{\subseteq}_{\mathfrak{n}}$ der Spinoperator des an dem Gitterplatz $\mathfrak{n}$ sitzenden Elektrons. Das Austauschintegral $I_{\mathfrak{n}, \mathfrak{m}}=I$ zwischen den Elektronen auf den Gitterplätzen $\mathfrak{n}$ und $\mathfrak{m}$ soll nur für nächste Nachbarn von Null verschieden sein und für alle übrigen Konfigu-

3 W. L. Bontsch-Brujewitsch, Fortschr. Phys. 3, 408 [1955]. 
rationen verschwinden. Insgesamt habe der betrachtete Kristall $2 N$ Atome.

In der Näherung der Energieschwerpunktmethode ${ }^{4}$ erhält man für die Energiezustände des betrachteten Systems aus (1)

$$
E=2 H \mu_{\mathrm{B}} \sum_{\mathfrak{n}} \Im_{\mathfrak{n}}^{z}-\frac{p I}{2 N}\left(\sum_{\mathfrak{n}} \Im_{\mathfrak{n}}^{z}\right)^{2}
$$

( $p$ bedeutet die Zahl der nächsten Nachbarn eines Atoms). Das bedeutet, daß alle Energiezustände, die zu einem bestimmten Eigenwert der $z$-Komponente des Gesamtspins gehören, auch die gleiche Energie haben. Setzt man für die Eigenwerte der $z$-Komponente des Gesamtspins

$$
S^{z}=\sum_{n} \Theta_{\mathfrak{n}}^{z}=-(N-n),
$$

so kann man die zugehörigen Energiezustände in der Form

$$
E(n)=-2 H \mu_{\mathrm{B}}(N-n)-\frac{k \Theta}{N}(N-n)^{2}
$$

schreiben, wenn man noch

$$
k \Theta=p I / 2
$$

setzt.

Jeder Zustand, der zu einem bestimmten $n$ gehört, ist $(2 N)$-fach entartet, denn zu $S=-(N-n)$ gibt es $(2 N)$ Eigenzustände des Hamilton-Operators (1), wenn man die Bedingung beachtet, daß diese auch Eigenzustände der Operatoren $\left(\Im^{z}\right)^{2}$ zum Eigenwert 1/4 sein müssen (pro Gitterplatz ist genau ein unkompensierter Spin vorhanden). In anschaulicher Formulierung kann man sagen, daß man, ausgehend von dem Zustand $S=-N$ auf $\left(\begin{array}{c}2 N \\ n\end{array}\right)$ verschiedene Arten die Spins an $n$ Gitterplätzen umkehren kann.

Wir sehen nun das betrachtete System als Mitglied einer kanonischen Gesamtheit sehr vieler gleichartiger Systeme an. Dann lautet die Zustandssumme

$$
Z=\sum_{n=0}^{2 N}\left(\begin{array}{c}
2 N \\
n
\end{array}\right) e^{2 \beta H \mu_{\mu}(N-n)+\beta(k \Theta / N)(N-n)^{2}} .
$$

\section{Einführung von FERMI-Quasiteilchen}

Es wird nun behauptet, daß man die Zustandssumme (3) in der Form

$$
Z=\sum_{n_{j r}=0,1} e^{-\beta E\left(n_{j r}\right)}
$$

${ }^{4} \mathrm{~S}$. W. Wonsowski, Die moderne Lehre vom Magnetismus, Deutscher Verlag der Wissenschaften, Berlin 1956. mit

$$
\begin{aligned}
& E\left(n_{j r}\right)=2 H \mu_{\mathrm{B}} \\
& \sum_{r=1}^{N}\left(n_{1 r}-n_{2 r}\right) \\
&-\frac{k \Theta}{N}\left[\sum_{r=1}^{N}\left(n_{1 r}-n_{2 r}\right)\right]^{2}
\end{aligned}
$$

schreiben kann.

Dabei wird summiert über alle möglichen Konfigurationen, in denen die Zahlen $n_{1 r}$ und $n_{2 r}$ unabhängig die Werte 0 und 1 annehmen können. Mit anderen Worten wird behauptet, daß man das betrachtete System auffassen kann als aus Quasiteilchen variabler Anzahl zusammengesetzt, die der Fermi-Statistik gehorchen. In engem Zusammenhang damit steht die schon früher ${ }^{1,2}$ festgestellte Tatsache, daß man die bei der strengeren Behandlung des Modells auftretenden „Spinwellen" als Quasiteilchen auffassen kann, die der Fermi-Statistik gehorchen ${ }^{5}$. Der Beweis für den hier behaupteten Sachverhalt kann durch eine einfache Abzählung geführt werden.

Dazu wird ein Zustand mit bestimmter $z$-Komponente des Gesamtspins betrachtet, d. h. mit bestimmtem $\sum_{r}\left(n_{1 r}-n_{2 r}\right)=N-n$. Man kann einen derart gekennzeichneten Zustand dadurch realisieren, daß man aus den $N$ Zahlen $n_{1 r}(r=1,2, \ldots, N)$ etwa $p$ Zahlen herausgreift, die 1 sind (die anderen sind Null) - dafür gibt es $\left(\begin{array}{l}N \\ p\end{array}\right)$ Möglichkeiten - , aus den $N$ Zahlen $n_{2 r}(r=1,2, \ldots, N) p-N+n$ Zahlen, die 1 sind (die restlichen sind wieder Null) dafür gibt es $\left(\begin{array}{c}N \\ p-N+n\end{array}\right)=\left(\begin{array}{c}N \\ 2 N-n-p\end{array}\right)$ Möglichkeiten. Insgesamt gibt es also

$$
g(N-n)=\sum_{p=0}^{N}\left(\begin{array}{l}
N \\
p
\end{array}\right)\left(\begin{array}{c}
N \\
2 N-n-p
\end{array}\right)=\left(\begin{array}{c}
2 N \\
n
\end{array}\right)
$$

Realisierungsmöglichkeiten. $g(N-n)$ ist aber genau das in (3) vorkommende statistische Gewicht des Zustands ${ }^{6} E(n)$.

Es ist klar, daß die hier angestellten Überlegungen automatisch auch für die der Quantentheorie angepaßten Form der Langevinschen Theorie des Paramagnetismus gelten, Spin $1 / 2$ pro Atom und kom-

5 Man vergleiche dazu Anm. 2. Dort ergab sich für die Energiezustände der linearen Kette

$$
E=-2 H \mu_{\mathrm{B}}\left(N-\sum_{k} n_{k}\right)-\frac{I}{n}\left(N-\sum_{k} n_{k}\right)^{2},
$$

wobei die $n_{k}$ Besetzungszahlen für die dort eingeführten Spinwellenzustände sind. Ersetzt man die Hälfte der $n_{k}$ durch $1-n_{k}^{\prime}$, so erhält man formal das gleiche Resultat wie in Gl. (5).

6 Vgl. Anmerkung S. 799. 
pensierte Bahnmomente vorausgesetzt. Der Fall, daß die Atome höhere Spinmomente haben, wird im Anhang behandelt, dabei zeigt sich, daß die dann auftretenden Quasiteilchen intermediären Statistiken gehorchen. Die Verallgemeinerung dieser Resultate für den Fall des Ferromagnetismus soll hier jedoch nicht durchgeführt werden.

$$
\begin{gathered}
\langle M\rangle=\mu_{\mathrm{B}}\left\langle\sum_{r}\left(n_{1 r}-n_{2 r}\right)\right\rangle, \quad(7) \quad\left\langle M^{2}\right\rangle=\mu_{\mathrm{B}}^{2}\left\langle\left[\sum_{r}\left(n_{1 r}-n_{2 r}\right)\right]^{2}\right\rangle, \\
U=\left\langle E\left(n_{j r}\right)\right\rangle=-2 H \mu_{\mathrm{B}}\left\langle\sum_{r}\left(n_{1 r}-n_{2 r}\right)\right\rangle \frac{k \Theta}{N}\left\langle\left[\sum_{r}\left(n_{1 r}-n_{2 r}\right)\right]^{2}\right\rangle .
\end{gathered}
$$

\section{Mittelwerte für die Besetzungszahlen und deren Produkte}

Berechnet man die thermodynamischen Mittelwerte der Magnetisierung, des Quadrats der Magnetisierung und der Energie aus (4), so erhält man einfach$$
\text { ren viltelwert von } A\left(n_{1 r}, n\right.
$$

Ganz allgemein wird dabei die Bezeichnung $\left\langle A\left(n_{1 r}, n_{2 r}\right)\right\rangle$ für den Mittelwert von $A\left(n_{1 r}, n_{2 r}\right)$ wie auch im folgenden immer benutzt:

$$
\left\langle A\left(n_{1 r}, n_{2 r}\right)\right\rangle=\sum_{n_{j r}} A\left(n_{1 r}, n_{2 r}\right) e^{-\beta E\left(n_{1 r}, n_{2 r}\right)} / \sum_{n_{j r}} e^{-\beta E\left(n_{1 r}, n_{2 r}\right)} .
$$

Zur Berechnung der interessierenden thermodynamischen Mittelwerte ist also in diesem Falle die Kenntnis der Mittelwerte $\left\langle n_{j r}\right\rangle$ und $\left\langle n_{j r}, n_{j^{\prime} r^{\prime}}\right\rangle$ erforderlich und ausreichend. Bei letzteren ist für $j=j^{\prime}$ wegen $n_{j r}{ }^{2}=n_{j r}$ im folgenden $r \neq r^{\prime}$ zu nehmen. Man erhält für diese Mittelwerte (dabei folgen wir in den Bezeichnungen im wesentlichen Kaschluhn ${ }^{7}$ )

$$
\left\langle n_{j r}\right\rangle=1 / 1+\frac{Z_{0}^{(j r)}}{Z_{1}^{(j r)}}, \quad(10) \quad\left\langle n_{j r} n_{j^{\prime} r^{\prime}}\right\rangle=\frac{Z_{11}^{(j r)\left(j^{\prime} r^{\prime}\right)}}{Z_{00}^{(j r)\left(j^{\prime} r^{\prime}\right)}+Z_{10}^{(j r)\left(j^{\prime} r^{\prime}\right)}+Z_{01}^{(j r)\left(j^{\prime} r^{\prime}\right)}+Z_{11}^{(j r)\left(j^{\prime} r^{\prime}\right)}} .
$$

Dabei ist

$Z_{0}^{(j r)}=\sum_{n_{i s}}^{(j r)} e^{-\beta E_{0}^{(j r)}}, Z_{1}^{(j r)}=\sum_{n_{i s}}^{(j r)} e^{-\beta E_{1}^{(j r)}}$

und

$Z_{\varrho \sigma}^{(j r)\left(j^{\prime} r^{\prime}\right)}=\sum_{n_{i s}}^{(j r)\left(j^{\prime} r^{\prime}\right)} e^{-\beta E_{\varrho \sigma}^{(j r)\left(j^{\prime} r^{\prime}\right)}}, \varrho, \sigma=0,1$.

$E_{0}^{(j r)}$ bedeutet $E\left(n_{1 r}, n_{2 r}\right)$ mit $n_{j r}=0, E_{1}^{(j r)}$ bedeutet $E\left(n_{1 r}, n_{2 r}\right)$ mit $n_{j r}=1$, der Index $(j r)$ an den entsprechenden Summenzeichen besagt, daß über $n_{j r}=0,1$ aussummiert worden ist. Entsprechend bedeutet $E_{\varrho \sigma}^{(j r)\left(j^{\prime} r^{\prime}\right)}$ in (13) $E\left(n_{1 r}, n_{2 r}\right)$ mit $n_{j r}=\varrho$ und $n_{j^{\prime} r^{\prime}}=\sigma$, der Index $(j r)\left(j^{\prime} r^{\prime}\right)$ am Summenzeichen besagt, daß über die betreffenden Besetzungszahlen aussummiert worden ist.

${ }^{6}$ Es sei noch ausdrücklich auf folgendes hingewiesen: Das hier benutzte Verfahren, durch das die Natur der Quasiteilchen bestimmt wurde, die das betrachtete System darzustellen gestatten, beruht im Grunde genommen auf einer Abzählung der Zustände des Systems. Wenn auch das Resultat, das man dabei erhält, nicht eindeutig zu sein braucht (man vergleiche dazu Anm. ${ }^{5}$ ), so kann man sicher in vielen Fällen durch ein Abzählverfahren wertvolle Hinweise auf die Natur der betreffenden Quasiteilchen be-
Wir berechnen zunächst die Mittelwerte der Besetzungszahlen. Da alle Zahlen $j, r$ für $r=1,2, \ldots, N$ gleichberechtigt sind, erhält man

$$
\left\langle n_{j r}\right\rangle=\frac{Z_{1}^{(j N)}}{Z_{0}^{(j N)}+Z_{1}^{(1 N)}}
$$

unabhängig von $r$. Ferner ist natürlich

$$
Z=Z_{0}^{(j r)}+Z_{1}^{(j r)}, r=1,2, \ldots, N
$$

Zur Berechnung von $Z_{0}^{(1 N)}$ überlegt man sich folgendes: In $Z_{0}^{(1 N)}$ wird über alle Zustände summiert, bei denen $n_{1 N}=0$ ist. Einen Zustand $E\left(n_{1 r}, n_{2 r}\right)$, der zu festem $\sum_{r}\left(n_{1 r}-n_{2 r}\right)=N-n$ gehört, kann man dadurch realisieren, daß man aus den $N-1$ Zahlen $n_{1 r}(r=1,2, \ldots, N-1) p$ herausgreift, die 1 sind, die übrigen sollen verschwinden, und aus den $N$

kommen. Wesentlich an dem hier behandelten Modell ist, $\mathrm{da} \beta$ es dieselbe Struktur wie das kompliziertere, durch den Diracschen Austauschoperator beschriebene hat, nicht bezüglich des Energiespektrums, sondern bezüglich der Basisvektoren, die den Hilbert-Raum aufspannen. Das erklärt, daß man in beiden Fällen Quasiteilchen, die der FERmi-Statistik gehorchen, zur Beschreibung des Systems verwenden kann.

7 F. Kaschluhn, Ann. Phys., Lpz. 16, 257 [1955]. 
Zahlen $n_{2 r} \quad(r=1,2, \ldots, N) p-N+n$, die restlichen sind wieder Null. Dafür hat man $\left(\begin{array}{c}N-1 \\ p\end{array}\right)$ bzw. $\left(\begin{array}{c}N \\ p-N+p\end{array}\right)=\left(\begin{array}{c}N \\ 2 N-p-n\end{array}\right)$ Möglichkeiten. Insgesamt kann man den betrachteten Zustand also auf

$$
\sum_{p=0}^{N-1}\left(\begin{array}{c}
N-1 \\
p
\end{array}\right)\left(\begin{array}{c}
N \\
2 N-p-n
\end{array}\right)=\left(\begin{array}{c}
2 N-1 \\
n-1
\end{array}\right)
$$

verschiedene Arten realisieren. Daher ist

$$
Z_{0}^{(1 N)}=\sum_{n=1}^{2 N}\left(\begin{array}{c}
2 N-1 \\
n-1
\end{array}\right) e^{-\beta E(n)},
$$

und wegen (15) erhält man sofort

$$
Z_{1}^{(1 N)}=\sum_{n=0}^{2 N-1}\left(\begin{array}{c}
2 N-1 \\
n
\end{array}\right) e^{-\beta E(n)} .
$$

Die Berechnung von $Z_{0}^{(2 N)}$ und $Z_{1}^{(2 N)}$ gestaltet sich analog. Man erhält

$$
Z_{0}^{(2 N)}=Z_{1}^{(1 N)}, Z_{1}^{(2 N)}=Z_{0}^{(1 N)} .
$$

Daraus folgt sofort

$$
\sum_{r=1}^{N}\left(\left\langle n_{1 r}\right\rangle+\left\langle n_{2 r}\right\rangle\right)=N,
$$

d. h., daß die mittlere Anzahl der Quasiteilchen fest ist, obwohl ihre Gesamtzahl in den einzelnen $\mathrm{Zu}$ ständen durchaus nicht fixiert ist.

Führt man noch ein

$$
F(\xi, \eta)=\sum_{n=0}^{2 N-1}\left(\begin{array}{c}
2 N-1 \\
n
\end{array}\right) e^{2 \xi\left(N-\frac{1}{2}-n\right)+(\eta / N)\left(N-\frac{1}{2}-n\right)^{2}},
$$

so kann man

$$
\begin{aligned}
& Z_{0}^{(1 N)}=Z_{1}^{(2 N)}=e^{-x+\frac{y}{4 N} F\left(x-\frac{y}{2 N}, y\right),} \\
& Z_{1}^{(1 N)}=Z_{0}^{(2 N)}=e^{x+\frac{y}{4 N}} F\left(x+\frac{y}{2 N}, y\right), \\
& \left\langle n_{1 r}\right\rangle=1 /\left\{1+e^{-2 x} \frac{F\left(x-\frac{y}{2 N}, y\right)}{F\left(x+\frac{y}{2 N}, y\right)}\right\} . \\
& \left\langle n_{2 r}\right\rangle=1 /\left\{1+e^{2 x} \begin{array}{r}
F\left(x+\frac{y}{2 N}, y\right) \\
F\left(x-\frac{y}{2 N}, y\right)
\end{array}\right\},
\end{aligned}
$$

schreiben mit $x=\beta H \mu_{\mathrm{B}}, y=\beta k \Theta$.

Die Berechnung von (11) gestaltet sich ganz ähnlich. Man erhält zunächst wegen der Gleichberechtigung aller Zahlen $j r(r=1,2, \ldots, N)$

$$
\begin{aligned}
\left\langle n_{j r} n_{j r^{\prime}}\right\rangle & =\frac{Z_{11}^{(j N)(j, N-1)}}{Z}\left(r \neq r^{\prime}\right) ;\left\langle n_{j r} n_{j r}\right\rangle=\left\langle n_{j r}\right\rangle \\
\left\langle n_{1 r} n_{1 r^{\prime}}\right\rangle & =\frac{Z_{11}^{(1 N)(2 N)}}{Z} .
\end{aligned}
$$

Mit Hilfe der zur Berechnung von $Z_{0}^{(j N)}$ angewandten Methode weist man nun nach, daß

$$
\begin{aligned}
& Z_{11}^{(1 N)(1, N-1)}=Z_{10}^{(1 N)(2 N)}=\sum_{n=0}^{2 N-2}\left(\begin{array}{c}
2 N-2 \\
n
\end{array}\right) e^{-\beta E(n)}, \\
& Z_{11}^{(2 N)(2, N-1)}=Z_{01}^{(1 N)(2 N)}=\sum_{n=2}^{2 N}\left(\begin{array}{c}
2 N-2 \\
n-2
\end{array}\right) e^{-\beta E(n)}, \\
& Z_{11}^{(1 N)(2 N)}=Z_{00}^{(1 N)(2 N)}=\sum_{n=1}^{2 N-1}\left(\begin{array}{c}
2 N-1 \\
n-1
\end{array}\right) e^{-\beta E(n)}
\end{aligned}
$$

ist. Daraus folgt sofort

$\left\langle n_{1 N} n_{1, N-1}\right\rangle+\left\langle n_{2 N} n_{2, N-1}\right\rangle+2\left\langle n_{1 N} n_{2 N}\right\rangle=1$.

Wegen (16), (18) und (19) ist nun ferner

$\sum_{r, r^{\prime}}\left\langle n_{1 r} n_{1 r^{\prime}}\right\rangle+\sum_{r r^{\prime}}\left\langle n_{2 r} n_{2 r^{\prime}}\right\rangle$

$$
=N^{2}-2 N(N-1)\left\langle n_{1 N} n_{2 N}\right\rangle,
$$

so daß wegen (8) und (9) zur Berechnung der uns interessierenden thermodynamischen Größen die Kenntnis von $\left\langle n_{1 N} n_{2 N}\right\rangle$ genügt. Unter Einführung von

$$
G(\xi, \eta)=\sum_{n=0}^{2 N-2}\left(\begin{array}{c}
2 N-2 \\
n
\end{array}\right) e^{2 \xi(N-1-n)+\frac{\eta}{N}(N-1-n)^{2}}
$$

kann man schreiben

$$
\begin{aligned}
& Z_{10}^{(1 N)(2 N)}=e^{2 x+\frac{y}{N}} G\left(x+\frac{y}{N}, y\right), \\
& Z_{00}^{(1 N)(2 N)}=Z_{11}^{(1 N)(2 N)}=G(x, y), \\
& Z_{01}^{(1 N)(2 N)}=e^{-2 x+\frac{y}{N}} G\left(x-\frac{y}{N}, y\right)
\end{aligned}
$$

mit $x=\beta H \mu_{\mathrm{B}}$ und $y=\beta k \Theta$ wie oben. Damit erhält man letzten Endes

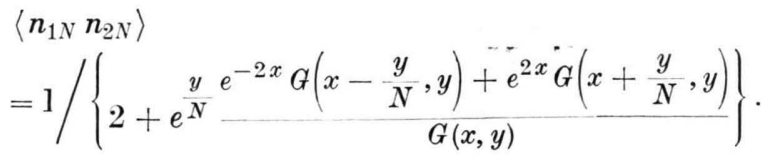

\section{Thermodynamische Mittelwerte für Magnetisie- rung, spontane Magnetisierung und Energie}

Die uns interessierenden thermodynamischen $\mathrm{Zu}$ standsgrößen des Systems sind nun leicht zu erhal- 
ten. Aus (7) und (17) ergibt sich für den Mittelwert der Magnetisierung

$M=\langle M\rangle$

$=2 N \mu_{\mathrm{B}} \frac{e^{x} F\left(x+\frac{y}{2 N}, y\right)-e^{-x} F\left(x-\frac{y}{2 N}, y\right)}{e^{x} F\left(x+\frac{y}{2 N}, y\right)+e^{x} F\left(x-\frac{y}{2 N}, y\right)}$,

Für den Mittelwert des Quadrats der Magnetisierung erhält man aus (8), (20) und (21) unter Vernachlässigung von Größen von der Ordnung $N$

$$
\left\langle M^{2}\right\rangle=4 N^{2} \mu_{B}^{2}\left[1-4\left\langle n_{1 N} n_{2 N}\right\rangle\right],
$$

während sich dann die mittlere Energie aus (9), (22) und (23) berechnen läßt:

$$
U=-H\langle M\rangle-\frac{k \Theta}{4 N^{2} \mu_{B}^{2}}\left\langle M^{2}\right\rangle .
$$

Man erkennt sofort - man hätte das natürlich auch unmittelbar aus der Zustandssumme ablesen können - , daß die mittlere Magnetisierung - in $\mathrm{Zu}-$ kunft einfach als Magnetisierung bezeichnet - für $H=0$ verschwindet.

Ihr relatives mittleres Schwankungsquadrat, gegeben durch

$$
s_{1}=\frac{\left\langle M^{2}\right\rangle}{M^{2}}-1
$$

wird daher - von der Feldstärke $H$ abhängig für $H=0$ sogar unendlich groß. Das bedeutet aber, daß $M$ keine für das Einzelsystem charakteristische Größe sein kann, wenigstens in der hier dargestellten Behandlung. Eine Bedeutung kann $M$ nur haben für die Beschreibung des mittleren Verhaltens einer Gesamtheit sehr vieler Systeme entsprechend der der kanonischen Gesamtheit zugrunde liegenden Idee, wobei aber das Einzelverhalten von System zu System großen Schwankungen unterworfen sein mag. Sicher aber kann man $M$ nicht dazu benutzen, um den Fernordnungsgrad - die für die Ferromagnetika so charakteristische Größe - zu kennzeichnen. Andererseits ist $M$ im Sinne von

$$
M=-\frac{\partial F}{\partial H} \quad \text { mit } \quad F=-k T \log Z
$$

konjugierte Zustandsgröße zu dem äußeren Magnetfeld $H$, was die oben eingeführte Bezeichnung rechtfertigt.

Zur Kennzeichnung des Fernordnungsgrads bietet sich dagegen $\left\langle M^{2}\right\rangle$ an. Das zugehörige mittlere Schwankungsquadrat, gegeben durch

$$
s_{2}=\frac{\left\langle M^{4}\right\rangle}{\left\langle M^{2}\right\rangle^{2}}-1
$$

und der Bequemlichkeit halber direkt berechnet aus

$$
s_{2}=\frac{4 N^{2} \mu_{B}^{2} T}{\left\langle M^{2}\right\rangle^{2}} \frac{\partial}{\partial \Theta}\left\langle M^{2}\right\rangle
$$

wird sich für den Fall, daß $\left\langle M^{2}\right\rangle$ merklich von Null verschieden ist, als sehr klein erweisen. Wir werden

$$
M_{\mathrm{sp}}=\sqrt{\left\langle M^{2}\right\rangle}
$$

als spontane Magnetisierung bezeichnen. Eine vernünftige Bedeutung hat dies zwar zunächst nur für den Fall $H=0$, da $\left\langle M^{2}\right\rangle$ ein Mittelwert über alle Einzelsysteme ist, die mit beliebiger Magnetisierung auftreten können, wenn auch mit verschiedener zugehöriger Wahrscheinlichkeit. Es wird sich jedoch zeigen, daß schon unverhältnismäßig kleine Felder $H$ die Wahrscheinlichkeit für das Auftreten von Systemen, bezogen auf die kanonische Gesamtheit, mit einer Magnetisierung antiparallel zu $H$ so stark herabdrücken, da $M_{\text {sp }}$ praktisch als spontane Magnetisierung des Einzelsystems auch bei allen Werten des äußeren Feldes $H$ angesehen werden kann. Man mag dieses Verhalten, das auch von Epstein ${ }^{8}$ in der Spinwellentheorie festgestellt worden ist, vielleicht folgendermaßen interpretieren. Die kanonische Gesamtheit mit ihren vielen Einzelsystemen ist nichts anderes als ein Abbild eines Festkörpers, der aus vielen magnetischen Elementarbereichen von fester Größe besteht. Die Mittelwerte über die kanonische Gesamtheit sind gerade die Größen, die man an diesem makroskopischen (natürlich sind auch die Einzelsysteme auch immer noch makroskopisch zu nennen) System messen kann. In diesem Sinne ist dann die Magnetisierung des großen Systems Null für $H=0$, da ja alle Zustände gleich wahrscheinlich sind, die eine Magnetisierung verschiedener Richtung, aber von gleichem Betrag haben. $M=M(H)$ wäre dann mit der Hysterese des realen Festkörpers zu vergleichen.

Es darf natürlich nicht behauptet werden, daß diese eben dargestellte Interpretation des hier benutzten Rechenverfahrens ein Abbild der physikalischen Wirklichkeit wäre, vielmehr kann sie nur den Wert einer Plausibilitätsbetrachtung haben, die die auftretenden Eigentümlichkeiten verständlich macht. Man weiß ja, daß die Dinge in Wirklichkeit erheblich komplizierter liegen. So haben die realen

8 P. S. Epstein, Phys. Rev. 41, 91 [1932]. 
magnetischen Elementarbezirke keine feste Größe, BLOCH-Wände und die magnetische Anisotropie spielen eine erhebliche Rolle, ebenso die äußere Oberfläche und Abweichungen vom realen Kristallbau. Im Gegenteil dürfte die Analogie, die hier besteht, rein zufälliger Natur sein ${ }^{9}$. Es sei hier noch auf einen Umstand ausdrücklich hingewiesen:

In der hier gebotenen Behandlung ist $M_{\mathrm{sp}}$ keine der üblicherweise in der Thermodynamik benutzten Zustandsgrößen, dagegen wohl $M$, man möchte sagen, etwas unberechtigt, da $M$ für das Einzelsystem direkt keine Bedeutung hat. Das ändert sich mit einem Schlag, wenn man, wie auch in ${ }^{11}$, die $\mathrm{Zu}$ standssumme nur etwa unter Benutzung der $\mathrm{Zu}$ stände konstruiert, für die die Magnetisierung größer oder gleich Null ist, $H=0$ vorausgesetzt. Dann ist $M \mathrm{zu} H$ konjugierte Zustandsgröße und gleichzeitig charakteristisch für das Einzelsystem. Dann ist auch $M^{2}=M_{\mathrm{sp}}^{2}$, es stehen ja nur Systeme mit gleicher Richtung der Magnetisierung zur Konkurrenz. Darauf soll jedoch nicht weiter eingegangen werden, es versteht sich von selbst, wie man vorzugehen hat.

\section{Genäherte Auswertung mit Hilfe der Molekular- feldmethode}

Hier soll nur skizziert werden, was bei der Auswertung nach der Molekularfeldmethode zu tun ist. Bekanntlich geht man dabei so vor, daß man das (oder die) größte(n) Glied(er) der Zustandssumme

$$
h(n)=\left(\begin{array}{c}
2 N \\
N+n
\end{array}\right) e^{2 n x+\frac{y}{N} n^{2}}
$$

nach

$$
\partial h(n) / \partial n=0
$$

aufsucht. Wie man mit Hilfe einer Art Sattelpunktsmethode (vgl. auch Anm. ${ }^{10}$ ) beweisen kann, sind diese Maxima im allgemeinen sehr steil, so daß dieses Vorgehen berechtigt ist. Explizit erhält man diese Extrema aus

$$
\sigma=\mathfrak{T} \mathfrak{g}(\sigma y+x) .
$$

Für $T<\Theta$ hat diese Gleichung drei Wurzeln, von denen zwei zu Maxima von $h(n)$ gehören. Sei $\sigma$ diejenige, die für $H=0$ positiv ist, dann bestimmt sie gleichzeitig die spontane Magnetisierung nach

9 Der Grund dafür dürfte darin zu suchen sein, daß in der kanonischen Gesamtheit eine schwache, nicht näher definnierte Wechselwirkung zwischen den in der Gesamtheit enthaltenen Systemen besteht, die ein sehr stark verzerr-

$$
\sigma=\mathfrak{T} \mathfrak{g} \sigma y .
$$

Da nun die in (22) und (23) eingehenden Ausdrücke $F(\xi, \eta)$ und $G(\xi, \eta)$ eine ähnliche Struktur haben wie $Z$, kann man sie nach derselben Methode behandeln. Man erhält für $T<\Theta(\Theta>0)$ unter Vernachlässigung der $H$-Abhängigkeit

$$
M_{\mathrm{sp}}=2 N \mu_{\mathrm{B}} \operatorname{Tg} \sigma \frac{\Theta}{T}
$$

und

$$
M=M_{\mathrm{sp}} \mathfrak{\tau} \mathfrak{g} \frac{M_{\mathrm{sp}} H}{k T}
$$

unter Vernachlässigung der Abhängigkeit der spontanen Magnetisierung von $H$. [Man berücksichtigt dabei, daß $h(n)$ zwei Maxima hat.] Für $T>\Theta$ (der Fall $\Theta<0$ ist darin enthalten) erhält man in der gleichen Näherung wie in der Werss schen Theorie für die paramagnetische Suszeptibilität

$$
\chi=\frac{2 N \mu_{B}^{2}}{k(T-\Theta)} .
$$

Damit erweist sich die Molekularfeldmethode zunächst als konsistentes Verfahren. Andererseits zeigt (27), daß schon sehr kleine Magnetfelder genügen, um das ganze große System praktisch homogen zu magnetisieren. Für die mittleren Schwankungsquadrate erhält man aus $(24),(25),(27)$ und (28) im Falle des Ferromagnetismus

$$
s_{1}=\frac{1}{\operatorname{Sin}^{2} \frac{M_{\mathrm{sp}} \bar{H}}{k T}}, \quad s_{2}=\frac{\sigma}{N} \frac{1}{\operatorname{Evo}^{2} \sigma y-y},
$$

womit die im vorigen Abschnitt ausgesprochenen Behauptungen belegt sind.

\section{Darstellung durch wechselwirkungsfreie Quasiteilchen}

In dem hier behandelten Falle erschwert die Wechselwirkung die Berechnung der thermodynamischen Zustandsgrößen nicht wesentlich. Gerade das fordert dazu heraus, zu untersuchen, welche Resultate man erhält, wenn man wechselwirkungsfreie Quasiteilchen zur genäherten Darstellung der Energieniveaus benutzt, und in welchem Temperaturbereich diese Resultate brauchbar sind. Dazu wird die Zustandssumme (3) in folgender Weise umgeformt:

tes Abbild der Wechselwirkung zwischen den realen ferromagnetischen Elementarbereichen darstellt.

10 W. Heisenberg, Z. Phys. 49, 619 [1928]. 


$$
\begin{aligned}
Z=\sum_{n=0}^{N}\left(\begin{array}{c}
2 N \\
n
\end{array}\right) e^{2 \beta H \mu \mathrm{B}(N-n)+\beta \frac{k \Theta}{N}(N-n)^{2}} & \\
& +\sum_{n=0}^{n-1}\left(\begin{array}{c}
2 N \\
n
\end{array}\right) e^{-2 \beta H \mu \mathrm{B}(N-n)+\beta \frac{k \Theta}{N}(N-n)^{2}} .
\end{aligned}
$$

Um die Wechselwirkung zu beseitigen, wird in den Exponenten zunächst $(N-n)^{2} / N$ durch $N-2 n$ ersetzt. Das ist für die (für $H=0$ ) tiefsten Niveaus, die in den beiden Anteilen von $Z$ zu $n=0$ gehören (es sei $\Theta>0$ ), unerheblich, wenn $n \ll N$ ist. Sodann wird in beiden Anteilen von $Z$ die Summation von $n=0$ bis $n=2 N$ erstreckt. Das läuft bei genügend kleinem $H$ auf die Einführung hoch gelegener fiktiver Niveaus hinaus, die das System in Wirklichkeit nicht besitzt; bei genügend tiefer Temperatur spielen diese jedoch keine wesentliche Rolle. Diese beiden Näherungen bedingen also die Beschränkung auf tiefe Temperaturen. Damit erhält man an Stelle von $Z$

$$
\bar{Z}=e^{-N \beta k \Theta} \sum_{n=0}^{2 N}\left(\begin{array}{c}
2 N \\
n
\end{array}\right)\left\{e^{2 \beta(k \Theta+H \mu \mathrm{B})(N-n)}+e^{-2 \beta(k \Theta-H \mu \mathrm{B})(N-n)}\right\},
$$

wenn man in dem zweiten Term von $Z$ nach Durchführung der Näherungen $n$ durch $2 N-n$ ersetzt. Nach den in Abschnitt 2 durchgeführten Überlegungen kann man nun dafür schreiben

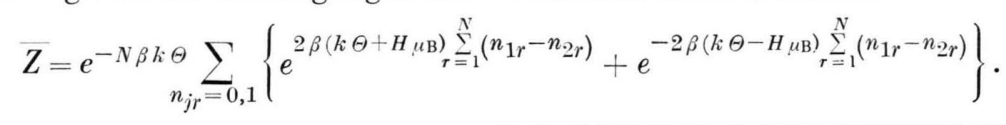

Es sei bemerkt, daß sich diese Darstellung nicht eindeutig aus $Z$ ergibt; die hier getroffene Wahl bedeutet, daß für $H=0$ die tiefsten Zustände des Systems gerade durch

$$
\begin{aligned}
& n_{1 r}=1 \text { und } n_{2 r}=0 \quad(r=1,2, \ldots, N) \\
& \text { bzw. } \quad n_{1 r}=0 \text { und } n_{2 r}=1 \quad(r=1,2, \ldots, N)
\end{aligned}
$$

erhalten werden. Das aber entspricht genau dem Verhalten der in Abschnitt 2 eingeführten Quasiteilchen.

Die Mittelwerte der Besetzungszahlen und ihrer Produkte werden wie in Abschnitt 3 berechnet. Man erhält für $H=0$

$$
\begin{aligned}
\left\langle n_{1 r}\right\rangle & =\left\langle n_{2 r}\right\rangle=\frac{1}{2}, \\
\left\langle n_{1 r} n_{2 r^{\prime}}\right\rangle & =\frac{1}{4{\left.\operatorname{Ev~}\right|^{2}}^{\frac{\Theta}{T}}}
\end{aligned}
$$

und damit für die spontane Magnetisierung

$$
M_{\mathrm{sp}}=2 N \mu_{\mathrm{B}} \widetilde{\tau} \mathfrak{g} \frac{\Theta}{T} .
$$

Man überzeugt sich davon, daß diese Näherung brauchbar ist für etwa $0 \leqq T<0,2 \Theta$. Die Mühe, die man für die Gewinnung dieser Resultate aufwenden muß, ist schon bei diesem einfachen Schulbeispiel verhältnismäßig gering. Andererseits stimmt dieses Ergebnis mit dem nach der Molekularfeldmethode erhaltenen überein, wenn man (26) mittels eines Iterationsverfahrens löst und sich mit dem ersten Schritt begnügt. In diesem Punkt weicht das

11 K. Meyer, Zur Theorie des Ferromagnetismus I, Z. Naturforschg. 12 a, 786 [1957], voranstehende Arbeit.
Verhalten des Systems von dem des Stonerschen Kollektivelektronenmodells ab (vgl. dazu Anm. ${ }^{11}$ ) . Es mag dies damit zusammenhängen, daß das hier betrachtete System bei $T=0^{\circ}$ magnetisch gesättigt ist, das Kollektivelektronenmodell dagegen im allgemeinen nicht.

Für $T>\Theta$ bietet die Methode der Elementaranregungen nicht das erwünschte Resultat, als man zwar das Curiesche Gesetz für die Suszeptibilität erhält, nicht jedoch das Curie-Weiss sche. Das mag darauf zurückzuführen sein, daß hier die Gesamtteilchenzahl nicht fixiert und infolgedessen das in ${ }^{11}$ verwendete Verfahren nicht anwendbar ist.

\section{Schlußbemerkungen}

Zum Schluß sei noch ein Punkt erwähnt, der bisher noch nicht zur Sprache gekommen ist. Bei der nach VAN VLECK ${ }^{12}$ gegebenen Begründung der WeIssschen Theorie wird so verfahren, daß man in dem Hamilton-Operator (1) einen der in dem von den $\Xi^{n}$ quadratisch abhängigen Teil stehenden Spinoperatoren direkt durch einen thermodynamischen Mittelwert ersetzt und dann damit in die statistische Rechnung eingeht. Dieses Verfahren hat insofern einen Schönheitsfehler, als es den eigentlichen physikalischen Untergrund der Theorie verschleiert mindestens in statistischer Hinsicht. Um es zum richtigen Resultat zu führen, müssen Zusatzvorschriften

12 J. H. van Vleck, Rev. Mod. Phys. 17, 27 [1945]. 
eingeführt werden, die nicht unmittelbar verständlich sind und nur durch den Erfolg bzw. durch die Kenntnis eines anderen Verfahrens gerechtfertigt werden.

Andererseits ist natürlich nichts dagegen einzuwenden, wenn eine Theorie des Ferromagnetismus dadurch begründet wird, daß Magnetisierung und Energie als Funktion von Temperatur und äußerem Magnetfeld vorgegeben werden. Eine solche Theorie wird zwar thermodynamisch konsequent durchführbar sein, aber immer phänomenologischen Charakter haben - nahegelegt durch gewisse Kenntnisse über die mikroskopische Beschaffenheit des Systems.

\section{Anhang \\ Über eine Interpretation der Langevinschen Theorie des Paramagnetismus}

Wir betrachten einen Kristall mit $2 N$ Atomen mit dem magnetischen Moment $\mu$, das bezüglich einer ausgezeichneten Achse, die durch die Richtung eines äußeren Magnetfeldes $H$ vorgegeben ist, $2 j+1$ Einstellungsmöglichkeiten hat $(j=$ Gesamtdrehimpulsquantenzahl, $\mu=\mu_{\mathrm{B}} g j, \mu_{\mathrm{B}}$ BoHrsches Magneton, g LandÉ-Faktor). Die Projektionen des magnetischen Moments auf diese ausgezeichnete Achse sind dann

$$
\mu \frac{j-n}{j}, \quad n=0,1, \ldots, 2 j .
$$

Damit sind die Energiezustände eines Atoms in einem äußeren Magnetfeld

$$
E=-H \mu\left(1-\frac{n}{j}\right)
$$

und die Zustandssumme für das System der $2 \mathrm{~N}$ Atome

$$
\left.Z=\left(\sum_{n=0}^{2 j} e^{\beta H \mu\left(1-\frac{n}{j}\right)}\right)\right)^{2 N} .
$$

$Z$ wird etwas umgeformt:

$$
Z=\left(\sum_{n=0}^{2 j} e^{-\beta H \mu \frac{n}{j}}\right)^{N}\left(\sum_{n=0}^{2 j} e^{\beta H \mu \frac{n}{j}}\right)^{N} .
$$

Führt man nun ein

$$
\begin{aligned}
& \varepsilon_{1}=-\frac{H \mu}{j}=-H \mu_{\mathrm{B}} g, \\
& \varepsilon_{2}=\frac{H \mu}{j}=H \mu_{\mathrm{B}} g,
\end{aligned}
$$

so erhält man die Zustandssumme in der Form

$$
Z=\left(\sum_{n=0}^{2 j} e^{-\beta \varepsilon_{1} n}\right)^{N}\left(\sum_{n=0}^{2 j} e^{-\beta \varepsilon_{2} n}\right)^{N} .
$$

Dies kann man so deuten, daß sich das System so verhält, als bestünde es aus Teilchen, die einer intermediären Statistik gehorchen, derart, daß jedes der Energieniveaus $\varepsilon_{1}$ und $\varepsilon_{2}$, die $N$-fach entartet sind, höchstens durch $2 j$ Teilchen besetzt werden kann. Im übrigen ist die Gesamtzahl dieser Teilchen nicht fixiert. Im Falle $j=\frac{1}{2}$ reduziert sich die intermediäre Statistik auf Fermi-Statistik.

Die mittleren Besetzungszahlen sind gegeben durch

$$
\left\langle n_{j}\right\rangle=-\frac{1}{\beta} \frac{\partial}{\partial \varepsilon_{j}} \log Z .
$$

Man rechnet leicht nach, daß

$$
\left\langle n_{1}\right\rangle+\left\langle n_{2}\right\rangle=2 j N,
$$

d. h. die mittlere Teilchenzahl ist konstant.

Das bedeutet, daß man die Langevinsche Theorie des Paramagnetismus (in ihrer der Quantentheorie angepaßten Form) ganz allgemein als eine sehr einfache "Spinwellentheorie“ interpretieren kann für Spinwellen, die einer intermediären Statistik gehorchen. Die maximale Besetzungszahl ist dabei durch die Drehimpulsquantenzahl pro Atom vorgeschrieben. Der Betrag des magnetischen Moments pro Spinwelle ist $g \mu_{\mathrm{B}}$, der Anregung einer Spinwelle im Zustand $\varepsilon_{1}$ entspricht dem Auftreten eines magnetischen Moments $g \mu_{\mathrm{B}}$ der einer Spinwelle im Zustand $\varepsilon_{2}$ dem Auftreten eines magnetischen Moments $-g \mu_{\mathrm{B}}$.

Herrn Professor Dr. G. Heber, Jena, sei an dieser Stelle für wertvolle Anregungen herzlich gedankt, ebenfalls zu danken habe ich Herrn Werner am Institut für theoretische Physik der Universität München für eine Diskussion über die hier behandelten Fragen. 